\title{
Personalie
}

Wolfgang G. Stock*

\section{Norbert Henrichs (1935-2016): Pionier der Informationswissenschaft in Deutschland}

DOI 10.1515/iwp-2016-0042

Zusammenfassung: Norbert Henrichs war ein bedeutender Pionier der Informationswissenschaft in Deutschland. Wir widmen uns der von ihm aufgebauten Philosophiedokumentation und der damit eng verbundenen Textwortmethode sowie einer frühen Form der informetrischen Themenanalyse. Herausgestellt wird seine führende Rolle beim Bemühen um eine Institutionalisierung der Informationswissenschaft, die allerdings mit der nachhaltigen Etablierung der Gesellschaft für Information und Dokumentation (GID) nicht gelang. In seinem Spätwerk hinterfragte Henrichs den kapitalistischen Informationsmarkt und stellt das „Menschsein im Informationszeitalter“ in den Fokus seiner Betrachtungen.

Deskriptoren: Norbert Henrichs, Informationswissenschaft, Philosophiedokumentation, Textwortmethode, Informetrie, Themenanalyse, Gesellschaft für Information und Dokumentation (GID), Informationsgesellschaft, Informationshermeneutik, Informationsanthropologie

\section{Norbert Henrichs (1935-2016): Pioneer of Information Science in Germany}

Abstract: Norbert Henrichs was an important pioneer of information science in Germany. We have a focus on his philosophy documentation and the closely associated text-word method as well as on an early form of informetric topic analyses. We emphasize his central role in attempts to institutionalize information science; however, the sustainable development of the Society for Information and Documentation (GID) failed. In his late work, Henrichs questioned the capitalist information market and highlights the "human existence in the information age".

Descriptors: Norbert Henrichs, Information science, Philosophy documentation, Text-word method, Informetrics, Topic analysis, Society for Information and Documentati-

*Kontaktperson: Wolfgang G. Stock, Henrich-Heine-Universität Düsseldorf, Abteilung für Informationswissenschaft,

E-Mail: stock@phil.hhu.de on (GID), Information society, Information hermeneutics, Information anthropology

\section{Norbert Henrichs (1935-2016): Pionnier de science de l'information en Allemagne}

Resumé:: Norbert Henrichs était un pionnier important de sciences de l'information en Allemagne. Nous regardons son documentation philosophique et la méthode du mot de texte associée et aussi une forme d'informetrie de sujets. Il avait un rôle central pour 1 institutionnalisation de la science de l'information, mais le développement de la Société de l'Information et de la Documentation (GID) ne réussit pas. In his late work, Henrichs questioned the capitalist information market and highlights the "human existence in the information age".

Descripteurs: Norbert Henrichs, Sciences de l'information, Documentation de philosophie, Méthode du mot de texts, Analyse de sujet, Société de l'Information et de la Documentation (GID), Société d'information society, Herméneutique d'information, Anthropologie d'information

Norbert Henrichs wurde am 5. Juli 1935 in Essen geboren; er starb am 2. Mai 2016 in Düsseldorf. Er war einer der bedeutendsten Informationswissenschaftler des 20. Jahrhunderts und gilt - neben Manfred Bonitz, Josef Koblitz, Hans-Werner Schober, Gernot Wersig, Harald H. Zimmermann und Rainer Kuhlen - als zukunftsweisender Pionier der deutschen Informationswissenschaft. Kuhlen (2014, v) nennt Henrichs einen „Gestalter der Informationswissenschaft“. Von 1967 bis zu seiner Emeritierung im Jahr 2001 war er zunächst wissenschaftlicher Mitarbeiter am Philosophischen Institut, dann Professor für Informationswissenschaft in Düsseldorf; zwischen 1980 und 1985 hatte er zusätzlich die Funktion des wissenschaftlichen Geschäftsführers der Gesellschaft für Information und Dokumentation (GID) in Frankfurt am Main inne.

Henrichs war vielseitig interessiert und auch in diversen Bereichen aktiv tätig. Durch sein Studium vorgegeben, publizierte er im Bereich von Theologie (z.B. Henrichs, 1967) und Philosophie, insbesondere zur Hermeneutik, der 
Lehre vom (richtigen) Verstehen (z. B. Henrichs, 1968). Zudem widmete er sich der Lokalgeschichte Düsseldorfs und des Stadtteils Kaiserswerth, wo er wohnte (z. B. Henrichs, Mayer, \& Menges, Hrsg., 1995). Er war aktiv in der Gemeinde seines Wohnortes und engagierte sich - zusammen mit seiner Frau - für sozial Benachteiligte (etwa Gefängnisinsassen) und bei Aktionen für die Dritte Welt (Vogels \& Wirtz, 2000). In der Flüchtlingsproblematik der letzten Jahre bezog er eine eindeutige Position, nämlich die auf der Seite von Pro Asyl. In diesem Bericht werden wir uns auf die Rolle Norbert Henrichs' als Informationswissenschaftler konzentrieren und die Zeitspanne von 1967, dem Beginn der Düsseldorfer Philosophiedokumentation (Abbildung 1), und 2014, dem Erscheinen seiner Ausgewählten Schriften (Henrichs, 2014) konzentrieren. Als besonders wertvolle Quelle liegt uns das Transkript eines Interviews vor, das im Rahmen von „Oral History“ im Jahr 2012 durchgeführt worden ist (Henrichs, 2012) (Abbildung 2). Zusätzlich berücksichtigen wir die Festschrift aus Anlass seines 65. Geburtstages (Schröder, Hrsg., 2000), einen Vortrag zur Geschichte der Informationswissenschaft, in dem Henrichs als Pionier der europäischen Informationswissenschaft gewürdigt wird (Hauk \& Stock, 2012), sowie Beiträge zur von Henrichs entwickelten Textwortmethode (Stock, 2000; Stock \& Stock, 2013, Kap. M.1). In der Festschrift befindet sich eine Bibliographie seiner informationswissenschaftlichen Schriften bis 1998 (Schröder, Hrsg., 2000, 365-371).

Zunächst werden wir auf die Düsseldorfer Philosophiedokumentation, das damit verbundene neue Erschließungsverfahren der Textwortmethode (Kapitel 1) sowie frühe Ansätze einer themenanalytischen Informetrie eingehen (Kapitel 2). Dann wenden wir uns der Institutionalisierung der Informationswissenschaft in Deutschland und insbesondere der Gesellschaft für Information und Dokumentation (GID) zu. Henrichs als Geschäftsführer der GID hat den Paradigmenwechsel der Informationspraxis in Deutschland hautnah miterlebt: den Übergang vom Staatsparadigma (der Staat sorgt für die Informationspraxis wie für eine öffentliche Infrastruktur) zum Marktparadigma (der Staat zieht sich zurück - auch aus der Finanzierung der GID - und überlässt die Informationspraxis dem kapitalistischen Markt) (Kapitel 3). Henrichs hat nie die Notwendigkeit eines Informationsmarktes negiert, aber er hat dessen Grundlagen und die Auswirkungen auf die Menschen nicht vergessen. „Nicht allein des Marktes wegen!“ ist eine vielbeachtete Publikation aus dem Jahr 1998 (in Henrichs, 2014, 144ff.). Henrichs“ Informationsanthropologie im Sinne des „Menschseins im Informationszeitalters" steht im Zentrum von Kapitel 4. Wir schließen den Artikel mit persönlichen Erinnerungen von Wegbegleitern von Norbert Henrichs.

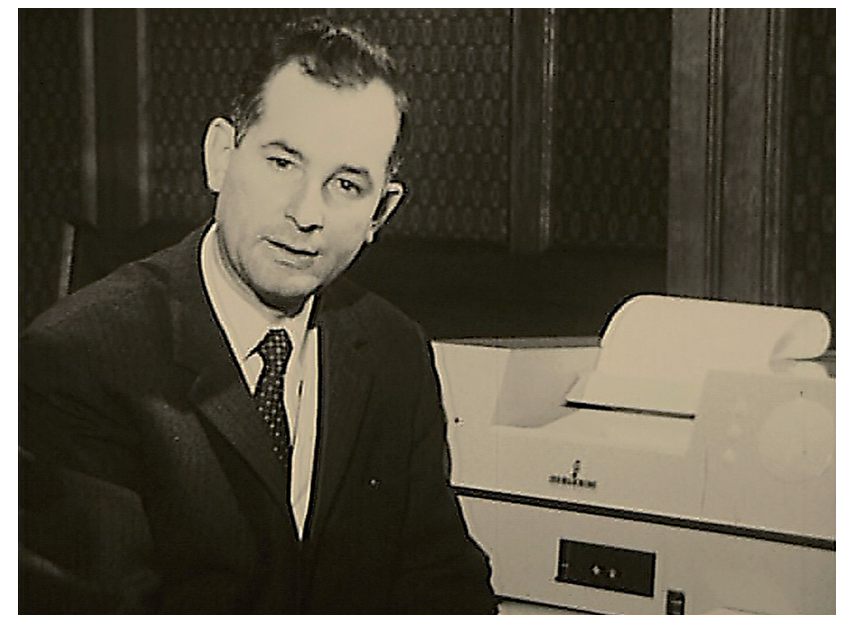

Abbildung 1: Korrespondenz mit dem Großrechner via Fernschreiber. Henrichs in einer Sendung des WDR im Jahr 1968. Quelle: WDR.

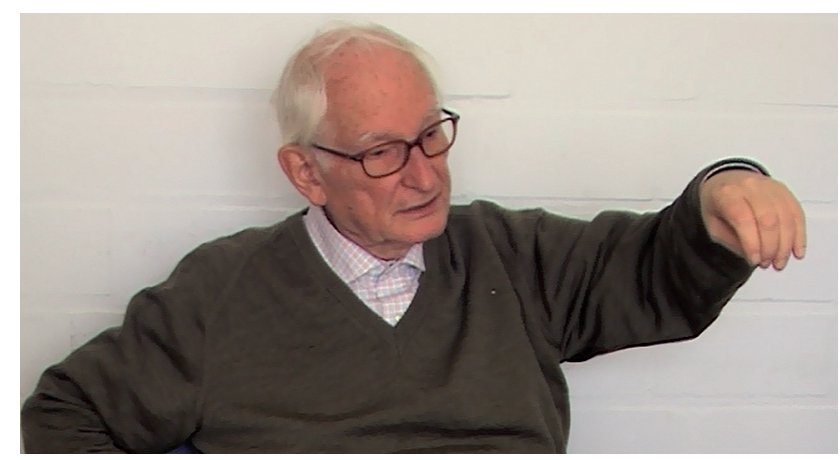

Abbildung 2: Henrichs im Oral-History-Interview an der Heinrich-Heine-Universität Düsseldorf im Jahr 2012. Foto: L. Beutelspacher.

\section{Philosophiedokumentation und Textwortmethode}

Im Jahr 1967 begann Henrichs ein Projekt zur philosophischen Dokumentation an der Universität Düsseldorf. Initiiert war das Vorhaben durch den Lehrstuhlinhaber der Düsseldorfer Philosophie, Alwin Diemer. Nicht zuletzt durch massive Werbung des Industriepartners (,Wenn Philosophen etwas nicht wissen, eine 4004 weiß es") war die Philosophiedokumentation ein Medienerfolg Ende der 1960er Jahre: der SPIEGEL berichtete (anonym, 1969), und der WDR gestaltete eine Fernsehsendung zum Thema (Abbildung 1). Allerdings war solch ein Unternehmen für die Bibliothekare und Archivare ein nahezu wahnwitziges Projekt. „Es gab damals einen unglaublichen Widerstand zu überwinden. Auch in den Bibliotheken. ... Die haben alle gesagt, was wir brauchen, gibt es nicht. Die Archivare, das war die andere Gruppe, die sagten das erst recht. Insofern ist das schon richtig mit dieser missionarischen 
Arbeit, die wir da betreiben mussten“ (Henrichs, 2012, 11). Übrigens hatte Henrichs einen frühen Besucher: Rainer Kuhlen hatte die Aufgabe, den Lehrgang für wissenschaftliche Dokumentare beim Lehrinstitut für Dokumentation (LID) neu zu gestalten. „Zur Vorbereitung dieser Reorganisation habe ich für ein paar Tage Henrichs in Düsseldorf besucht und einiges im Zusammenhang von GOLEM und PASSAT und der Kontroverse freies und kontrolliertes Vokabular etc. gelernt“ (R. Kuhlen, persönliche Mitteilung, 29.5.2016).

Henrichs diskutierte Klassifikation, Thesaurus und digitale Volltextspeicherung als Indexierungsmethoden, die er allesamt für die Zwecke der Philosophie verwarf. Klassifikationssysteme kamen nicht infrage, weil sie sowohl zu starr als auch ideologisch vorbelastet sind. Insbesondere die Hierarchierelation bereitete ihm Probleme: „Und dann dieses streng Hierarchische. Vielleicht ist es ja auch ein Merkmal der früheren Generation, der Gehorsam, der richtet sich ja auf eine Monospitze, und dann gliedert sich von da aus die Welt auf“ (Henrichs, 2012, 20). Beim Thesaurus störte Henrichs das dort verwendete kontrollierte Vokabular. „Wenn man einen Text erschließt, hat man den Thesaurus daneben, liest einen Sachverhalt, versteht ihn, so wie man ihn versteht, und sagt, das ist doch das“ (Henrichs, 2012, 20). Die Indexierung hängt somit wesentlich von den Ausdrucksmöglichkeiten des Vokabulars und vom Verständnis des Indexers ab, und das kann irregeleitet sein. Die Volltextverarbeitung wurde abgelehnt, weil sie zwar für Textstatistik sinnvoll sei, aber ansonsten im Retrieval zu ungeheurem Ballast führe.

Stattdessen setzte er auf eine textorientierte Methode der intellektuellen Inhaltserschließung („Stichwortabstracts“). Die Bezeichnung „Textwortmethode“ kam in den frühen Zeiten der Philosophiedokumentation noch nicht vor und wurde erst Jahre später verwendet. Die erarbeiteten Dokumentationseinheiten (genannt „Zielinformationen“) wurden über Lochstreifen in ein Computersystem eingelesen. Realisiert wurde die Datenbank von Siemens mit seinem System GOLEM. Der Zugriff auf die Datenbank erfolgte zunächst via Fernschreiber (Abbildung 1) und später dann über eine Standleitung zum Rechenzentrum. Henrichs betonte im Interview, dass sein System eine der ersten Datenbankanwendungen überhaupt war (Henrichs, 2012, 7). Gerard Salton, der der Philosophiedokumentation in Düsseldorf einen Besuch abstattete, zeigte sich zwar von der technischen Ausstattung begeistert, allerdings nicht vom Konzept. Im Gegensatz zu Henrichs, der auf menschliche Indexierung der Dokumente als Zwischenschritt der ansonsten maschinellen Dokumentation setzte, verfolgte Salton von Anfang an ausschließlich die automatische Indexierung.
Henrichs arbeitete bei der Philosophiedokumentation mit mehreren internationalen Partnern zusammen. Zu erwähnen sind insbesondere die Kooperationen mit dem Philosophy Documentation Center der Bowling Green State University in Ohio (mit Richard Lineback) sowie mit der Forschungsstelle und dem Dokumentationszentrum für Österreichische Philosophie in Graz (mit Rudolf Haller und Wolfgang L. Gombocz).

Wie arbeitet die Textwortmethode? Im Gegensatz zu Wissensordnungen wie Thesauri oder Klassifikationen, die stets mit Begriffen arbeiten, verwendet die Textwortmethode ausschließlich Wörter. Der Indexer ist gehalten, sich ausschließlich am empirisch vorliegenden Textmaterial zu orientieren. Das heißt, dass alle Textwörter originalsprachig und in der jeweiligen Orthographie des Autors erschlossen werden. So bleiben z.B. die Bedeutungsvarianten von „Sein“, „Seyn“, „Existenz“ und „Ek-sistenz“ bei Heidegger erhalten. Erlaubt sind lediglich grammatikalische Normierungen der Textwörter in ihre Grundform. Ausnahmen sind die Normansetzungen bei den historischen Bezügen, das sind Namen von Autoren, philosophischen Schulen oder Epochen.

Henrichs' philosophische Dokumentation war in den ersten Jahren zeitschriftenbasiert. Die technische Basis war das System ALBUM, wie GOLEM ein Produkt von Siemens. Eine philosophische Zeitschrift, ggf. auch eine thematisch ähnliche, auf einander folgende Reihe von Zeitschriften (wie beispielsweise „Annalen der Philosophie“, „Erkenntnis“ und „Journal of Unified Science“), wurde artikelweise von Band 1 bis zum Einstellen des Periodikums oder bis zum aktuellen Rand bearbeitet. Aufgenommen wurden die bibliographischen Angaben sowie die nach der Textwortmethode erschlossenen Inhalte der Dokumente. Die Register waren getrennt nach den historischen Bezügen (mit den Normeinträgen der Namen) und der Sachwörter, also der Textwörter. Wir wollen dieses Vorgehen mit einem Beispiel illustrieren. Unser Informationsbedürfnis sei die Diskussion zwischen Otto Neurath und Rudolf Carnap zum methodischen Solipsismus in der Zeitschrift „Erkenntnis“. Im Sachwortverzeichnis zur Zeitschrift „Erkenntnis“ (Belke, 1973) findet man den Eintrag „Solipsismus, methodischer“ (Abbildung 3). Untergeordnet sind alle Textwörter, die mit „Solipsismus, methodischer" in mindestens einer thematischen Kette in einem Artikel gemeinsam auftauchen, also „Aussage“, „Carnap, Rudolf“, „Einheitswissenschaft“ usw. bis „Zurückführung“. Die Nummern verweisen auf die Artikel in der Bibliographie. Die Treffer für „Carnap“ sind die Nummern 247 und 262, für „Neurath“ Nummer 263. Abbildung 4 zeigt die beiden Treffer 262 und 263. Es handelt sich um die Aufsätze „Protokollsätze“ von Neurath und 
„Über Protokollsätze“ von Carnap, beide aus dem Jahrgang 1932/33.

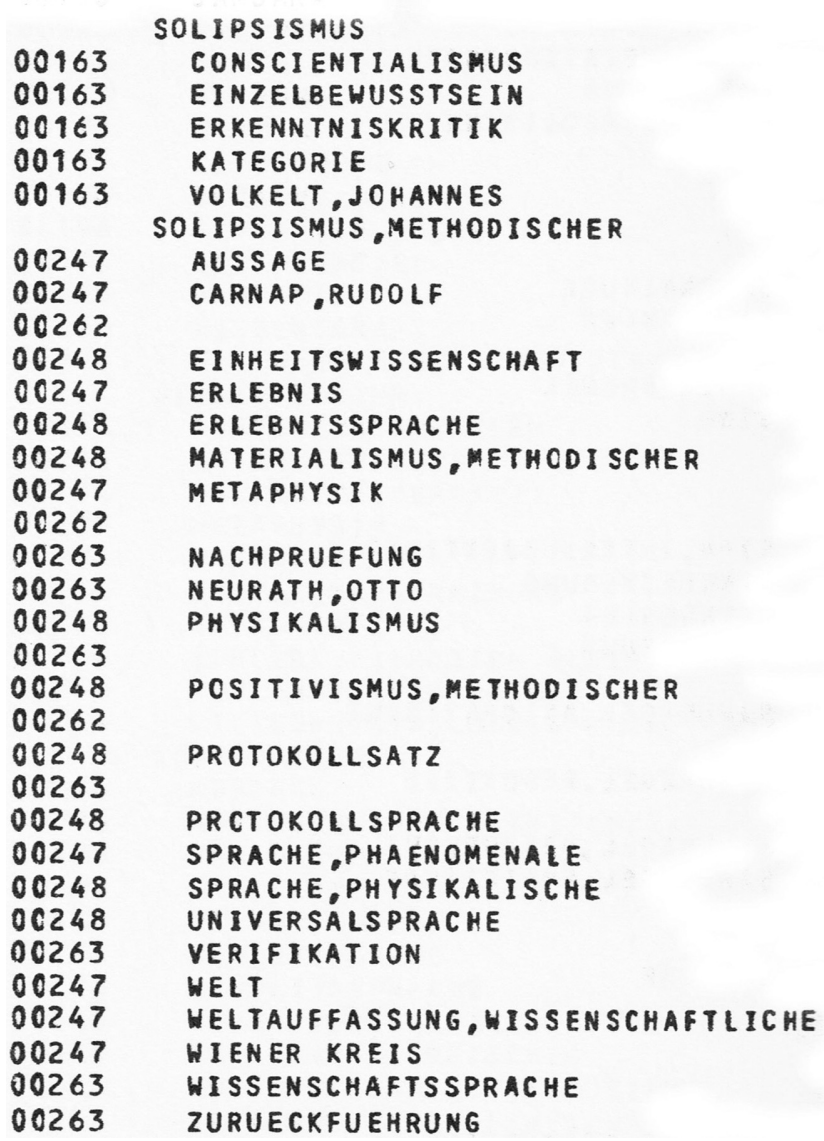

Abbildung 3: Sachwortverzeichnis zur Zeitschrift „Erkenntnis“. Quelle: Belke, 1973, 612.

Schauen wir uns das Dokument von Neurath (Nr. 262) genauer an! Insgesamt enthält das Indexat zehn thematischen Ketten. Als historische Bezüge sind „Carnap, Rudolf“ (in den drei Themenketten 5, 7 und 9) und „Wiener Kreis“ (Kette 10) genannt; alle anderen Einträge sind Sachwörter. Neuraths Text beginnt mit „Einheitssprache“ - „Einheitswissenschaft“ - „Protokollsatz“ - „Trivialsprache“ „Trivialsprache, physikalistische“ - „Physikalismus, radikaler“ - „Tautologie“ - „Universalslang“ - „Protokollsprache“, „Realsatz“, „Nichtprotokollsatz“ und „Satz“ (Kette 1) und endet mit „Wiener Kreis“ - „Einheitswissenschaft“ (Kette 10). Im Online-Retrieval waren Textwörter wie Themenketten suchbar. Während der Boolesche UND-Operator eine Dokumentationseinheit findet, wenn die mit UND verbundenen Suchatome überhaupt vorkommen (im Beispiel 262 beispielsweise „Protokollsatz UND Idealsprache“), findet der Abstandsoperator (heute meist SAME genannt) nur dann das Dokument, wenn die Suchatome auch in derselben Themenkette vorkommen. „Protokollsatz SAME Ideal- sprache" führt also - korrekterweise - nicht zum Treffer Nr. 262. Auf der Grundlage des syntaktischen Indexierens durch Themenketten wird eine recht ballastarme Recherche durchgeführt, die zu sehr guter Precision führt. Im Interview betonte Henrichs, dass die Textwortmethode ihre Stärke nicht nur durch die Verwendung des textspezifischen Termmaterials findet, sondern besonders durch das Gruppieren individueller Terme zu komplexen Themenketten (Henrichs, 2012, 25).

Henrichs strebte an, für alle Indexate in der Datenbank auch die Volltexte bereitzuhalten. Das technische Mittel seiner Wahl war der Mikrofilm als Partner des Computers (Abbildung 5). Der Industriepartner war diesmal die Fa. 3M. Henrichs“ Grundidee war, „neben dem Computer spielte der Mikrofilm eine große Rolle, Literatur zu verbreiten“ (Henrichs, 2012, 9).

Der Kombination aus gedrucktem Register und Volltexten in Mikroform blieb Henrichs in einem weiteren Großprojekt treu. Der gesamte publizierte Briefwechsel deutscher Philosophen aus einer „Glanzzeit“ der Philosophie (1750-1850) wurde erfasst und verfilmt (Henrichs \& Weeland, 1987; 1990). Die Volltexte füllen 3.141 Microfiches mit insgesamt 187.500 Textseiten; enthalten sind ca. 13.000 Einzelbriefe und rund 1.000 Briefsammlungen. „Der Nutzer der Briefdokumentation kann sich jetzt nicht nur rasch über die Fundorte publizierter Briefe bestimmter Autoren unterrichten, sondern erhält mit dieser Microfiche-Ausgabe nun auch direkten Zugang zu den Brieftexten und zur Umgebung ihrer Erstveröffentlichung, ohne den oft langen und zeitraubenden Weg über den bibliothekarischen Leihverkehr beschreiten zu müssen“ (Henrichs \& Weeland, 1990, 9).

Durch den textsprachlichen Ansatz wird sehr viel Sucharbeit auf den Nutzer verschoben. Er muss fremdsprachliche Äquivalente seines Sucharguments kennen und auch Vorkenntnisse über die sprachlichen Eigenarten bestimmter Autoren (wie Heideggers „Ek-sistenz“) haben. Henrichs schreibt: „Zu Wort kommt ausschließlich der jeweilige Textautor, assoziationsinitiativ sei ausschließlich der Benutzer“ (Henrichs, 1973, IX). Allerdings kann der Nutzer bei diesen Assoziationen unterstützt werden (Henrichs, 2012, 25). Dies geschah durch diverse Register, die nicht nur Retrievalhilfsmittel waren, sondern eine frühe Form der Informetrie darstellen. 


\section{BAND \\ JAHRGANG \\ VERFASSER \\ TITEL U SEITE \\ HIST. BEZUEGE \\ SACHWOERTER}

3

$1932 / 1933$

NEURATH, OTTO

PROTOKOLLSAETZE. 204-214

CARNAP, RUDOLF $(5,7,9) /$ WIENER KREIS (10)

EINHEITSSPRACHE $(1-3,5,8,10) /$ EINHEITSWISSENSCHAFT $(1,5,10) /$ PROTOKOLLSATZ (1,5-8)/ IDEALSPRACHE (2)/ TRIVIALSPRACHE (1-3)/

BALLUNG (3)/ TRIVIALSPRACHE, PHYSIKALISTISCHE (1-3)/

PHYSIKALISMUS, RADIKALER $(1,4)$ / TAUTOLOGIE (1)/ UNIVERSALSLANG

$(1-4,6,8) /$ METAPHYSIK $(4-5,9) /$ PROTOKOLLSPRACHE $(1,5,8) /$

ERLEBNISSPRACHE (5)/ REALSATZ (1)/ NICHTPROTOKOLLSATZ $(1,5) /$

PERSONENNAME (6)/ BEWAEHRUNG (7-8)/ REAKTIONSPRUEFUNG ( 8 )/

ZEICHENREIHE (8)/ SATZ $(1,7-8)$ / SOLIPSISMUS, METHODISCHER ( 9$)$ / POSITIVISMUS, METHODISCHER (9)

00263

\section{BAND \\ JAHRGANG \\ VERFASSER \\ TITEL U SEITE \\ HIST. BEZUEGE \\ SACHWOERTER}

3

$1932 / 1933$

CARNAP, RUDOLF

UEBER PROTOKOLLSAETZE.215-228

NEURATH, OTTO $(2,7) /$ POPPER, KARL RAIMUND (6-7)

PROTOKOLLSATZ $(1-4,7) /$ NACHPRUEFUNG $(1,5-6) /$ VERIFIKATION

$(1,5) /$ PHYSIKALISMUS (2)/ SYSTEMSPRACHE (3-5)/ SYSTEMSATZ

$(3-5) /$ SIGNAL (4)/ UEBERSETZUNGSREGEL (3-4)/ AUSSAGE $(3-4) /$

SATZ (3) / PROTOKOLLSPRACHE (3)/ PROTOKOLLAUSSAGE (3-4) /

UEBERSETZUNG $(3-4)$ / NATURGESETZ (5-6)/ SATZ, KONKRETER $(3,5)$ /

HYPOTHESE (5-6)/ SATZ, ABGELEITETER (3,5)/ PRIVATSPRACHE (4)/

ZURUECKFUEHRUNG $(1,3) /$ WAHRNEHMUNGSSATZ $(3,5) /$

SOLIPSISMUS, METHODISCHER $(1-2) /$ ABSOLUTISMUS (7)/

POSITIVISMUS, LOGISTISCHER (7)/ WISSENSCHAFTSSPRACHE (1-7)

Abbildung 4: Dokumentationseinheiten („Zielinformationen“) zur Zeitschrift „Erkenntnis“. Quelle: Belke, 1973, 67.

\section{Themenanalytische informetri- sche Untersuchungen}

Ziele der informetrischen Bemühungen Henrichs‘ waren einerseits das Erkennen von „Wissenskomplexen“ und andererseits das Verfolgen terminologischer Variationen im Laufe der Zeit. Eingesetzte Methoden umfassten Clusteranalysen zur Bestimmung von Zusammenhängen zwischen Textwörtern als Bezeichnungen der Wissenskomplexe und die Errechnung von Gewichtungswerten für Textwörter, um einen quantitativen Ausdruck für ein Thema zu einem bestimmten Zeitpunkt zu erhalten. „Das angewandte Verfahren erwies sich im weiteren Verlauf der methodischen Untersuchungen und der Entwicklung von Gewichtungsalgorithmen für Deskriptorenkomplexe als nicht nur hermeneutisch, sondern auch als heuristisch fruchtbar für wissenschaftssoziologische Fragestellungen. So kann z.B. an Ergebnissen retrospektiver Recherchen rechnergestützt argumentativer Wandel bei der Behandlung gegebener Themen nachgewiesen und dargestellt werden“ (Henrichs, 1990, 1071). Hauptanliegen aller informetrischen Analysen Henrichs` war jedoch nicht, empiri- sche Wissenschaftsforschung (oder in diesem Fall wohl besser: empirische Philosophieforschung; Stock, 1985) zu betreiben, sondern den Nutzer bei der Recherche assoziativ zu unterstützen (Henrichs, 2012, 25). Henrichs‘ Forschungen zur Informetrie fallen in die Jahre zwischen 1974 und 1980 (der Term „Informetrie“ wurde übrigens erst 1979 geprägt), um dann im Jahr 1990 noch einmal den „Begriffswandel in Datenbanken " zu thematisieren.

Verschiedene Formen einfacher Listen erleichtern das Auffinden guter Sucheinstiege. Die Palette reicht von alphabetisch sortierten Listen (Freiheit, geschöpfliche; Freiheit, göttliche; Freiheit, mathematische) über rückläufig sortierte alphabetische Listen (Ideologiefreiheit; Wahlfreiheit; Entscheidungsfreiheit) und permutiert sortierte Listen (Freie Arbeit; Freie Forschung; Freie Konkurrenz) bis hin zum Wortfragmentwörterbuch durch eine Zerlegung der Textwörter in Pentagramme (Erkennen von freih im Textwort Widerspruchsfreiheitsbeweis) (Henrichs, 2014, 333336). Das „Defizit“ der textsprachlichen Auswertung ist für Henrichs damit behoben. Im Rückblick auf diese Arbeiten sagte er, ,aus den Indexaten sind eine Fülle verschiedener Wörterbücher hervorgegangen: die rein klassische alphabetische Darstellung, dann die permutierte, mit den Adjek- 


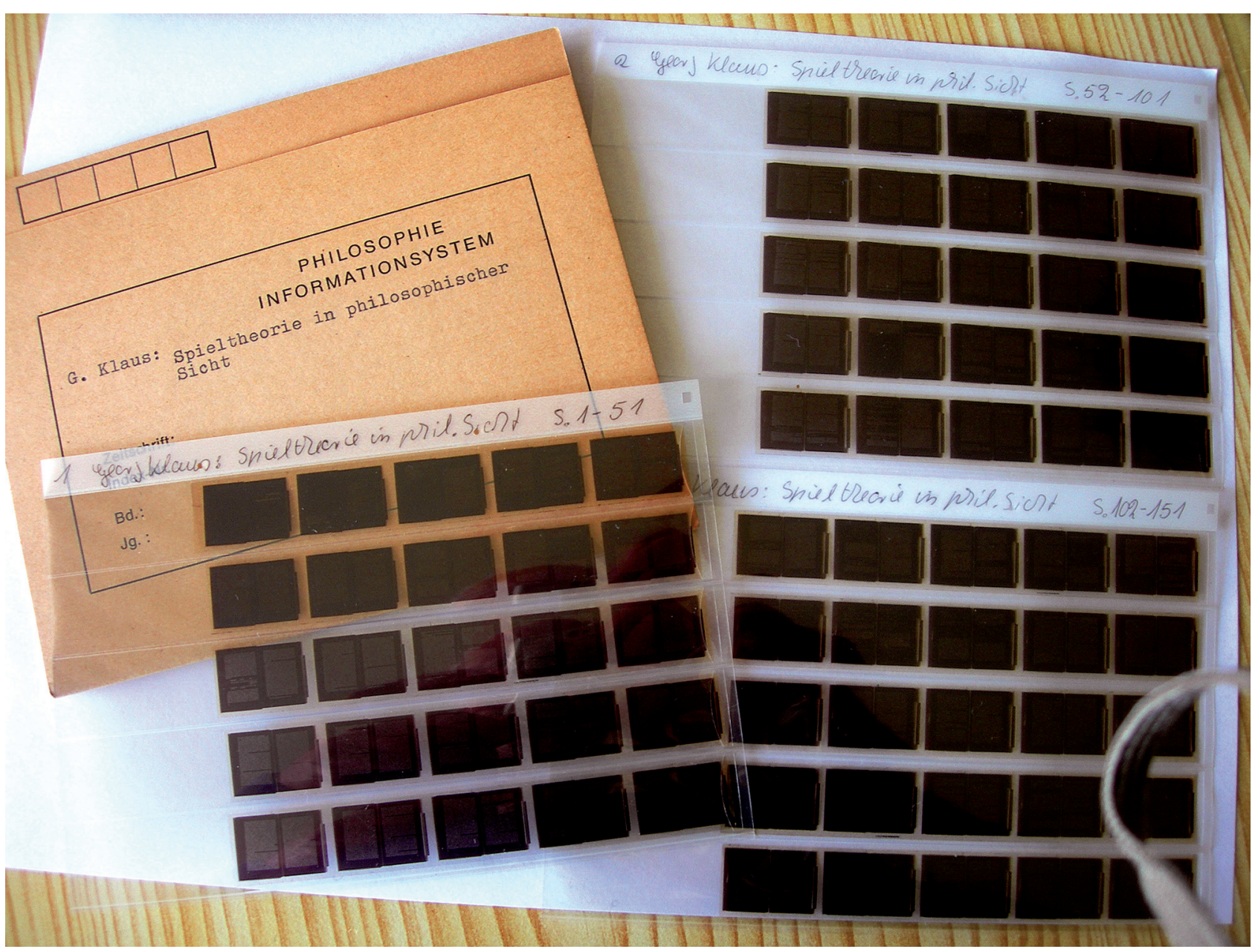

Abbildung 5: Der Mikrofilm als Volltextspeicher (Foto: W.G. Stock).

tiven nach vorne, eine rückwärts sortierte und dahinter die Fragmentmethode. Damit haben wir ja nun das Wortmaterial aufgeschlüsselt“" (Henrichs, 2012, 23). Henrichs' Pentagramme sind ein Vorläufer des n-Gramm-Retrievals, bei dem Wörter durch Zeichenfolgen mit der Länge $n$ (bei Henrichs war $n=5$ ) ersetzt werden. Mit seinen rückläufig sortierten Listen erreichte Henrichs eine problemlose Linkstrunkierung - ein Verfahren, das auch heute noch nur die wenigsten Retrievalsysteme beherrschen.

Eine einfache Form der Erstellung von Wissenskomplexen ist die Auflösung der thematischen Ketten in Zweierpaare, wie dies bereits in den Registern der ZeitschriftenDokumentation zu finden ist. Henrichs $(2014,321)$ gibt ein Beispiel für die „thematischen Bezüge“:

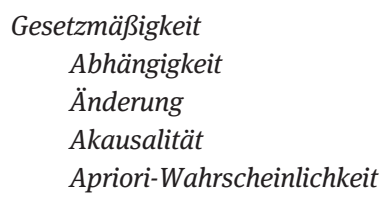

\section{Bedingtheit \\ Beobachtung}

„Die eingerückten Wörter zeigen ... den thematischen $\mathrm{Zu}$ sammenhang des Leitwortes an und vermitteln so über die Ausbreitung seines Verwendungszusammenhangs eine Übersicht über seine Bedeutungsdimensionen“ (Henrichs, 2014, 321). Die „thematischen Invarianten“ berücksichtigen Tripel und entstehen durch die Schnittmengenbildung der zugehörigen Indexketten, z. B. (Henrichs, 2014, 337):

Absolute,das
Arbeit
Autonomie
Bewusstsein
Denken
Descartes, Rene

„Dieses Wörterbuch ist für den Fragesteller besonders wichtig, weil es auf - möglicherweise latente - Zusammen- 
hänge von Sachwörtern über dritte Begriffe aufmerksam macht“ (Henrichs, 2014, 338). Ein Textwort ist in einem Dokument mehr oder weniger wichtig, je nachdem, in wie vielen und in welchen thematischen Ketten es vorkommt. Henrichs $(2014,345)$ berücksichtigte bei der Berechnung eines dokumentspezifischen Gewichtungswertes den „Deskriptorwert“ (die Anzahl der Ketten, in denen ein Textwort vorkommt), den „Indexkettenwert“ (die Mächtigkeit der Indexketten, in denen das Textwort auftritt) sowie den „Dokumentwert“ (die Summe der Indexkettenwerte, normiert auf 100) (Henrichs, 2014, 345-347). Die Gewichtungswerte gestatten ein gewichtetes Retrieval sowie ein Relevance Ranking der Treffer (letzteres wurde allerdings nicht technisch realisiert).

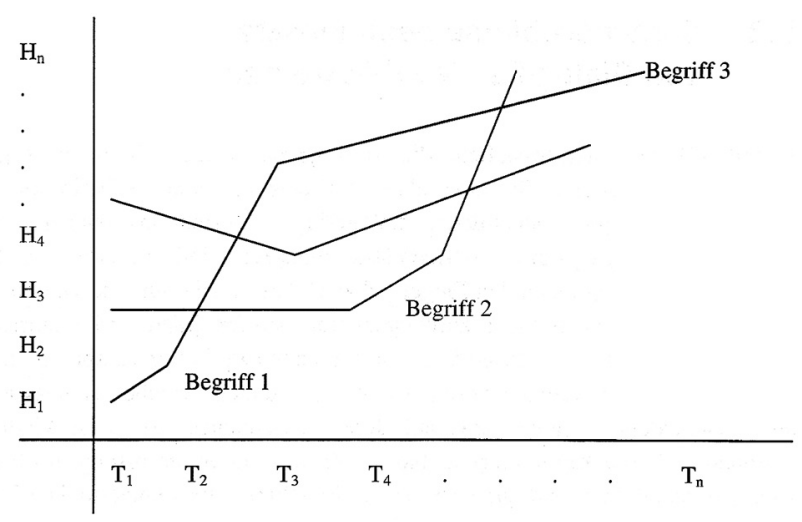

Abbildung 6: Informetrische Themenanalysen als Basis der Ideengeschichte. Quelle: Henrichs, 2014, 325. H: (relative) Häufigkeit; T: Zeit.

Man kann die dokumentspezifischen Gewichtungswerte einzelner Textwörter auch aggregieren, z.B. summieren und durch ihre Anzahl teilen. Wir erhalten so das durchschnittliche Gewicht eines Textwortes bezogen auf die jeweilige Dokumentmenge (etwa eines Jahrgangs). Eine graphische Aufbereitung einer solchen Zeitreihe zeigt Abbildung 6 am Beispiel dreier Textwörter. (Henrichs' Terminologie ist mitunter etwas ungenau. Es geht hier nicht um Begriffe und an anderen Stellen nicht um Deskriptoren im Sinne eines Thesaurus', sondern stets um Wörter.) „Dabei zeigt sich dann etwa, dass zu bestimmten Zeiten in Zusammenhang mit einem Thema (z. B. Freiheit) bestimmte andere Begriffe eine mehr oder weniger zentrale Rolle gespielt haben und die Bedeutung bestimmter Begriffe beeinflussten" (Henrichs, 2014, 325). Im Zusammenspiel der Wissenskomplexe und der zeitlichen Entwicklung ihrer Teile (also der einzelnen Textwörter) ist es möglich, semantische Konsistenzen bzw. Inkonsistenzen von spezifischen Termen zu eruieren. Über die genannten themenanalytischen Verfahren lassen sich ,ideengeschichtliche
Analysen“ (Henrichs, 2014, 349) empirisch starten. Für Henrichs sind solche informetrischen Analysen allerdings lediglich „heuristische Verfahren“. Für ihn kommt es bei der Informetrie nicht auf die Deutung solcher Verteilungen an, denn dies ist „Sache der Forschung“ (Henrichs, 2014, 325).

Die Philosophiedokumentation und auch die damit verbundenen themenanalytischen Studien wurden in Düsseldorf ca. 1990 vor allem aus Geld- und Personalmangel „immer mehr vernachlässigt“ und weitgehend eingestellt (Henrichs, 2012, 7). Mit dem Aufkommen der Folksonomies in den Social Media sah Henrichs allerdings eine Option, die Indexierung im Rahmen der Textwortmethode durch die Nutzer vornehmen zu lassen. „Ja ... durch Kooperation. Das wäre eine Möglichkeit. Diese Methoden-Verfeinerung lässt sich nur machen auf dieser humanen Basis. Wenn alle anpacken“ (Henrichs, 2012, 29). Ihm schwebte offenbar ein gemischt intellektuell-automatisch arbeitendes Verfahren vor. Der Nutzer ist für das Markieren der wichtigen Textwörter zuständig; alles andere erledigt die Maschine. Insbesondere das syntaktische Indexieren dürfte für Laien wenig durchschaubar sein. Hier sollte man die Absätze der Fachartikel herannehmen und durchnummerieren. Textwörter, die im gleichen Absatz stehen, erhalten somit dieselbe Kettennummer. „Wörter, die man aus einem Absatz nimmt, stehen in hoher Wahrscheinlichkeit in einem thematischen Zusammenhang. ... Wenn wir einen Aufsatz schreiben und bringen einen neuen Gedanken, (dann fangen) wir einen neuen Absatz (an)“ (Henrichs, 2012, 26). Mit der Verbindung zwischen Textwortmethode und Social Tagging könnte m. E. ein interessanter Forschungsbereich entstehen, der näher untersucht gehört.

\section{Das luD-Programm und die GID}

Das IuD-Programm, an dessen Realisierung Henrichs mitgearbeitet hat, sah den Aufbau bzw. die Weiterführung von 16 disziplinspezifischen Fachinformationszentren (z.B. FIZ 4 Energie, Physik, Mathematik) sowie von vier weiteren querschnittsorientierten Fachinformationszentren (z. B. für Patente) sowie die Zusammenführung bereits vom Bund geförderter Einrichtungen zur Gesellschaft für Information und Dokumentation (GID) in Frankfurt am Main vor (BMFT, 1974, 43 ff.).

„Wir haben unendlich oft getagt“, sagte Henrichs (2012, 33), „und die Idee war, ein großes zentralistisch organisiertes System zu haben“. Vorbilder waren u.a. Institutionen wie das Zentralinstitut für Information und Dokumentation (ZIID) in der DDR. Es ging allerdings nicht 
darum, eine Monostruktur zu haben. „Das Ministerium hat sich schon als Spitze verstanden, aber darunter gab es dann sofort eine Verbreitung. Es gab sechshundertsoundsoviel Dokumentationsstellen in Deutschland und die wurden jetzt auf zwanzig reduziert bzw. ... zur Kooperation gezwungen“ (Henrichs, 2012, 33). Solch ein neu organisierter Kranz von Informationssystemen und -zentren braucht eine zentrale Infrastruktureinrichtung, „die Entwicklung macht, die auch technisch unterstützt. Die sozusagen, wie die Spinne im Netz, Dienstleistungen erbringt für die Fachinformationseinrichtungen“ (Henrichs, 2012, 34).

Henrichs konnte mit der Sektion für Kommunikation (SfK, Leiter zwischen 1982 und 1984: Wolf Rauch) bei der GID nur einen einzigen Forschungs- und Entwicklungsbereich neu schaffen. Ansonsten wurden u. a. folgende „alte“ Einrichtungen in die GID überführt:

- Institut für Dokumentationswesen (IDW; gegründet 1962)

- Zentralstelle für Maschinelle Dokumentation (ZMD; gegründet 1964)

- Studiengruppe für Systemforschung (SfS, gegründet 1959)

- Abteilung für Nichtnumerik am Deutschen Rechenzentrum (letzteres 1961 gegründet)

- Dokumentationszentrum für Informationswissenschaft (ZDok; gegründet 1971, vorher seit 1962 die Bibliothek und Dokumentationsstelle der Deutschen Gesellschaft für Dokumentation)

Die GID kam damit auf rund 300 Mitarbeiter und einen Jahresetat von 25 Millionen DM. Rückblickend bezeichnete Henrichs diese Zusammenfassung von fünf Einrichtungen unter ein Dach als „Geburtsfehler der ganzen Geschichte von vorneherein" (Henrichs, 2012, 35). Nachdem der zunächst berufene wissenschaftliche Geschäftsführer, Norbert Szyperski, nach wenigen Wochen abgesagt hatte und zur Gesellschaft für Mathematik und Datenverarbeitung (GMD) wechselte, war Henrichs der Wunschkandidat des damaligen Bundesministeriums für Forschung und Technologie (BMFT). Er trat seine Stelle im Jahr 1980 an. Obgleich er auf fünf Jahre von der Universität Düsseldorf beurlaubt war, hat Henrichs seine gesamte Lehre (jeweils montags von früh bis spät) weitergeführt. Nach Marlies Ockenfeld (2016, 159) stellt dieser Lebensabschnitt Henrichs' ein „berufliches Intermezzo“ dar. Sein Lebensmittelpunkt war nämlich stets Düsseldorf und die von ihm aufgebaute Düsseldorfer Spielart der Informationswissenschaft.

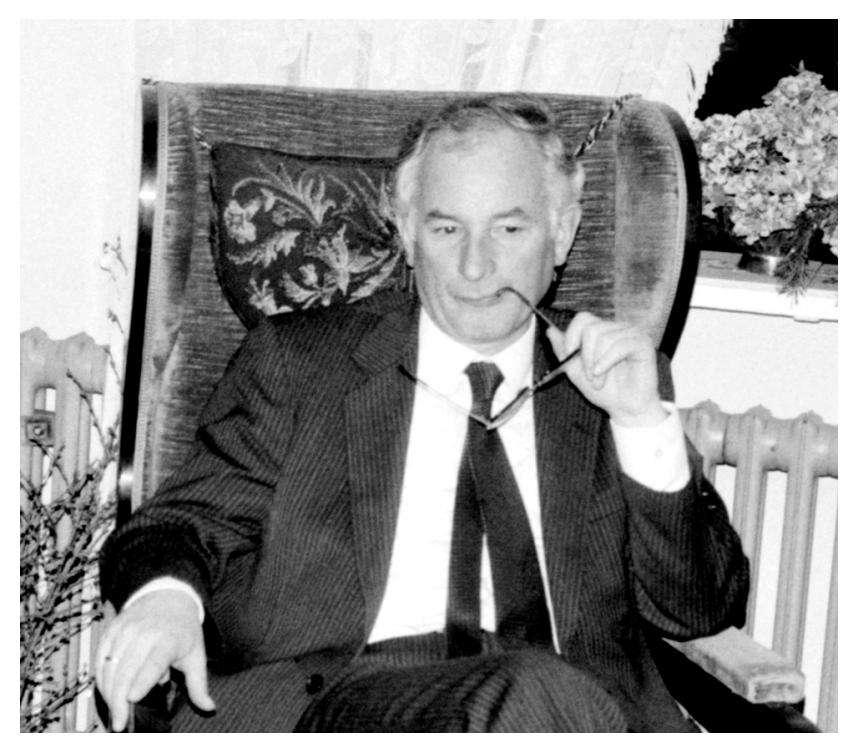

Abbildung 7: Henrichs zu seiner Zeit als wissenschaftlicher Geschäftsführer der GID (1982) (Foto: M. Kirschbaum).

Für Wolf Rauch (2000, 25) war es „eine bewegte Zeit an der GID, in der wir in einem Zweifrontenkrieg gegen das aufkommende Marktparadigma einerseits und die Trägheit verkrusteter Strukturen andererseits ankämpfen mußten“. Die verkrusteten Strukturen fanden eine ihrer Ursachen in den übernommenen vormals eigenständigen Institutionen. Die Sektionsleiter regierten ihre Bereiche „wie kleine Kurfürsten“, wie dies der SPIEGEL (anonym, 1987, 43) ausdrückt: „An Zusammenarbeit schien niemand interessiert“. Die GID hatte drei mächtige Betriebsräte. „Eigentlich hätte man die Institute, die existierten, zumachen müssen und dann neu anfangen. Das ging aber nicht, weil die Personalverträge einfach dem entgegenstanden“" (Henrichs, 2012, 43). Der Chef des Rechenzentrums beispielsweise „hat das nie überwunden, dass er da integriert werden musste und sich einem Geschäftsführer, einem wissenschaftlichen Geschäftsführer, unterwerfen musste“ (Henrichs, 2012, 36).

Die zweite Ursache dürfte im Verhalten der Ministerialbürokratie beim Ministerium für Forschung und Technologie gelegen haben. Der SPIEGEL berichtete, „Ministerialbeamte aus Bonn mischten sich ständig über seinen (d.h., Henrichs') Kopf hinweg in die Forschungsarbeit ein. Mitarbeiter bekamen unmittelbar aus Bonn Direktiven freilich gewöhnlich mit ständig anderen, widersprüchlichen Vorgaben“ (anonym, 1987, 43). Insbesondere das IDW hatte als Projektträger des BMFT seinen direkten Draht nach Bonn. „Das IDW blieb eigentlich immer ein bisschen (außen) vor. Das war diese Fördereinrichtung. Die arbeitete für den BMFT, war aber bei uns angegliedert“ (Henrichs, 2012, 36). 
Der SPIEGEL macht auf einen dritten Aspekt aufmerksam: die „mangelnde Kompetenz“ einiger Mitarbeiter, die zudem „überholten Fragestellungen“ nachgingen (anonym, 1987, 43). Insbesondere der letzte Grund veranlasste den Wissenschaftsrat (1984) zu hinterfragen, ob die GID die Fördervoraussetzungen überhaupt erfülle. Henrichs $(2012,43)$ betonte im Interview, dass die GID-Mitarbeiter von der technischen Entwicklung überrollt worden waren. Es kamen die PCs, aber die GID war auf Großrechneranwendungen konzentriert. „Das waren alles Entwicklungsgeschichten, und da war die GID überflüssig“. Insbesondere im Rechenzentrum waren „die Leute zu alt“ und „es stimmte die Ausrichtung nicht mehr“ (Henrichs, 2012, 42). Henrichs war offenbar von einigen seiner Mitarbeiter enttäuscht, wenn diese nicht die geringste Flexibilität zeigten. „Mir haben die Leute immer, wenn ich die Qualifikation ansprach, ihre Bewerbungsanzeige (gezeigt), auf die sie sich gemeldet haben - ,Biologe gesucht stand dann da“. Außerdem war es „einfach nicht möglich, hier das Personal aufzufrischen" (Henrichs, 2012, 37 f.). Durch Finanzierungslücken und andere Gründe wurden viele der ursprünglich geplanten FIZ nicht realisiert, was der GID Aufgaben wegnahm. „Als die Planung fertig war, da ging dem BMFT der Saft aus. Da kam die Ölkrise und da war plötzlich kein Geld mehr da“ (Henrichs, 2012, 34).

Rainer Kuhlen (persönliche Mitteilung, 30.5.2016) berichtet rückblickend über das Scheitern der GID: „Die Auflösung der GID stand nicht zuletzt auch im Zusammenhang mit dem schon seit 1980 einsetzendem Rückzug der staatlichen Förderung der Informationswissenschaft (sowohl der Wissenschaft als auch der Infrastruktur). Dagegen hatten die GID und auch Norbert Henrichs, vielleicht auch wegen ihrer nicht überzeugenden Wissenschaftsleistung und angesichts der fehlenden Unterstützung der Fachinformationszentren, kaum eine Chance“. Im Fachinformationsprogramm 1990-1994 wird eine klare Leitlinie zugunsten des Marktparadigmas vorgegeben. „Herstellung und Angebot von Produkten und Dienstleistungen im Markt sind eine originäre Aufgabe privatwirtschaftlicher Unternehmen; sie müssen eigenverantwortlich entscheiden, in welchem Umfang sie Fachinformationen produzieren und anbieten wollen.... Die Bundesregierung hält deswegen an ihrer Ansicht fest, keinen Einfluß auf Produktions- und Angebotsstrukturen zu nehmen" (BMFT, 1990, 20, 22). Damit wird das Staatsparadigma der Lechmann-Thesen (Henrichs, 2007) und des IuD-Programms endgültig verabschiedet. Henrichs selbst hat die GID im Jahr 1985 verlassen und ihre Abwicklung (im Jahr 1987) nicht mehr als Geschäftsführer verantwortet.

Noch immer liegen Materialien zum IWD und der ZDM sowie zu Kommissionen der DGD - unbearbeitet - im Ar- chiv der Düsseldorfer Informationswissenschaft. Da ,sind die ganzen Planungsunterlagen“ (Henrichs, 2012, 59); „ich habe alle Planungsakten aus Frankfurt mitgenommen“ (Henrichs, 2012, 33).

\section{Informationsanthropologie}

Insbesondere bedingt durch das Scheitern der GID und dem Richtungswechsel in Richtung Markt widmete sich Henrichs ab Ende der 1980er Jahre - allerdings in kritischer Distanz - dem Informationsmarkt. In einem Interview mit Willi Bredemeier betonte Henrichs, „natürlich geben wir dem Markt sein Recht. Aber wir denken auch darüber nach, die Informationsgesellschaft menschlicher zu machen" (Henrichs, 2000, 35).

Was heißt für Henrichs „Informationsgesellschaft“? Dies ist eine Gesellschaft, „die Information (jeder Art) als wichtigen Rohstoff erkennt und bewertet“ (Henrichs, 2014, 109). Es geht in dieser Gesellschaft nicht primär um „Informatisierung“ (also um Technikeinsatz), sondern um eine „informierte Gesellschaft, die ein ausgeprägtes „Informations(wert)bewusstsein“ an den Tag legt (Henrichs, 2014, 62). Verlässt man sich ausschließlich auf den Markt, droht die Gefahr einer „Informations-Klassengesellschaft“ (Henrichs, 2014, 59) mit „Informationskrösussen“ auf der einen Seite und „Informationshabenichtsen“ auf der anderen (Henrichs, 2014, 101). Auch setzt man die Gesellschaftsmitglieder der Macht verschiedener Unternehmen aus, etwa der Chiphersteller, der Hard- und Softwaremarktführer, der Betreiber von Rechenzentren und Netzen sowie der Datenbankhersteller, -anbieter und -vertreiber (Henrichs, 2014, 142). Schon im Jahr 1990 (also lange vor Google oder Facebook) wies Henrichs auf die „Verdatung“ der Gesellschaftsmitglieder hin. „Wer seiner persönlichen Verdatung prinzipiell Widerstand entgegensetzt, fällt aus der wirtschaftlichen, sozialen, medizinischen oder kulturellen Versorgung weitgehend heraus" (Henrichs, 2014, 141). Auch eine andere Prognose Henrichs' ist eingetroffen. „Die für den Betrieb von Informationssystemen außerordentlich hohen Vorleistungen begünstigen nicht selten Monopolbildungen auf der Anbieterseite“ (Henrichs, 2014, 140). Vor allem bedingt durch Netzwerkeffekte, gibt es in der Tat auf Informationsmärkten eine Tendenz zur Monopolisierung (Linde \& Stock, 2011) - man denke nur an die herausragenden (Macht-)Positionen von Google, Facebook, eBay oder Amazon.

Setzt man ausschließlich auf den Markt, droht „Kapitalismus pur“ und man setzt sich „einer um sich greifenden Ökonomisierung unserer gesamten Lebenswelt“ aus (Henrichs, 2014, 150). Dagegen setzte Henrichs auf die 
Zukunftsverträglichkeit des Informationsmarktes: „Das neue Paradigma muss demnach lauten: Zukunftssicherung vor kurzfristigem Marktnutzen" (Henrichs, 2014, 153). Zur Zukunftssicherung gehört auch das Erreichen oder Erhalten von Frieden. Hier schloss sich Henrichs dem MitSchöpfer der Dezimalklassifikation und Friedensnobelpreisträger Henri Lafontaine an. „Voraussetzung für den Weltfrieden ist, dass die Menschen sich verstehen. Damit sie sich verstehen, müssen sie wissen, was sie denken und wissen. Was sie denken und wissen, kann man nicht zuletzt in den Büchern und sonstigen Schriften der Menschen nachlesen. Infolgedessen muss man ihre Schriften sammeln, erschließen, nachweisen und zugänglich machen, um das Verstehen der Menschen untereinander voranzubringen“ (Henrichs, 2014, 163f.) - kurz: Man muss Wissensorganisation betreiben.
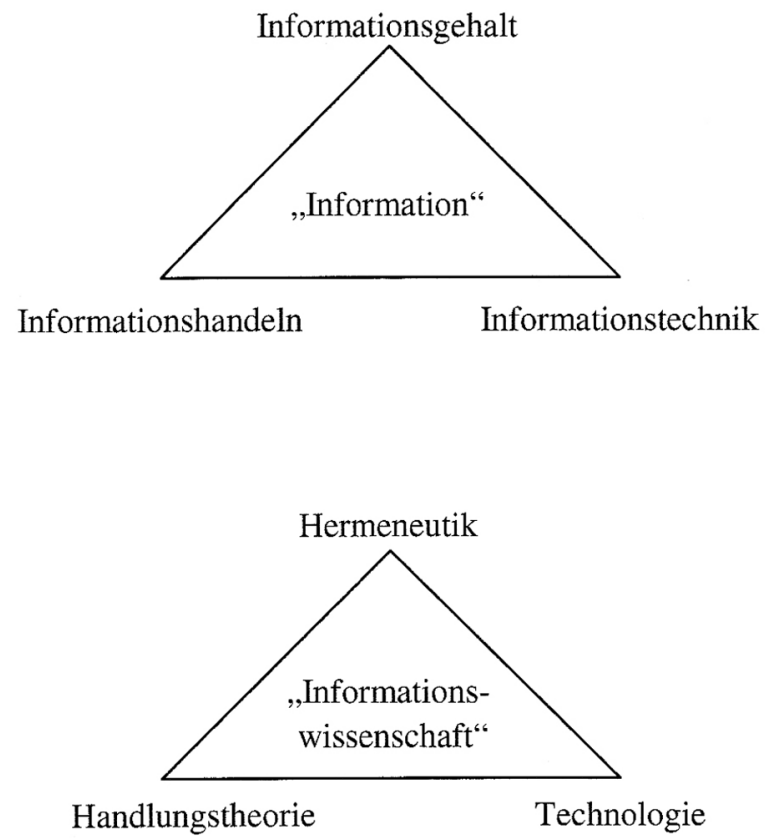

Abbildung 8: Modelle von „Information“ und „Informationswissenschaft“ nach Henrichs. Quelle: Henrichs, 2014, 176.

Wie definierte Henrichs unsere Disziplin? Informationswissenschaft ist „die Wissenschaft von der Wissensorganisation“ (Henrichs, 2012, 17), genauer der „Wissensverwertungsorganisation“ (Henrichs, 2014, 193). Grundbegriff ist „Information“ (Abbildung 8 oben), der sich triadisch in den Informationsgehalt (also das Wissen), das Umgehen mit der Information („Informationshandeln") und in die Informationstechnik aufspaltet. Wenn wir Wissen organisieren, müssen wir dieses verstehen. Damit gehört die Informationshermeneutik (Abbildung 8 unten) auf jedem Fall zur Informationswissen- schaft. „Der Mensch ist prinzipiell ein verstehendes Wesen. Wenn wir durch die Welt laufen, interpretieren wir immer sofort. Einiges konstruieren wir da hinein" (Henrichs, 2012, 17f.). Dem menschlichen Informationshandeln widmet sich die informationswissenschaftliche Handlungstheorie. Diesem pragmatischen Aspekt wies Henrichs als „Humanfaktor“ die primäre Aufgabe der Informationswissenschaft zu (Henrichs, 2014, 177). Dagegen erscheint die Technologie eher nebensächlich.

„Die Bibliothekare tun das (Wissensorganisation betreiben), indem sie Bücher sammeln und Kataloge machen. Die Archivare machen das auf ihre Weise und die Dokumentare (auch). Die Dokumentare sind eigentlich aus Kritik an der Unzulänglichkeit der Wissensaufbereitung durch die anderen entstanden" (Henrichs, 2012, 18). Wie sah Henrichs seine eigene Rolle bei der Entwicklung der Informationswissenschaft? Er verortete sich bei zwei Aspekten. Der erste betrifft die Institutionalisierung der Informationswissenschaft: „Politikberatung war meine wichtigste Aufgabe“ (Henrichs, 2012, 45). Der zweite Aspekt beschreibt den Entstehungsweg der Informationswissenschaft. „Wenn ich auf das Lebenswerk zurück schaue und mir auf der anderen Seite klar mache, welche Entwicklung wir in der Zwischenzeit gehabt haben, dann kann ich nur sagen, wir haben nicht mehr, aber auch nicht weniger geleistet, als einen Wagen mit sehr holprigen Rädern und schlechter Konstruktion über eine sumpfige Wiese zu schieben bis an den Rand der asphaltierten Straße. Und dann kam auf einmal der große Durchbruch“ (Henrichs, 2012, 31): PCs und Smartphones wurden zu Gebrauchsgegenständen, Wissen wird aus riesigen Datenbanken recherchiert und Nutzer beteiligen sich in den Social Media mit Informationsproduktion und der Indexierung (durch „Taggen“) ihrer Dokumente. Sind Dokumentation und Informationswissenschaft jetzt überflüssig? Geben wir die Wissensorganisation wieder an die Bibliothekare und Archivare zurück und übergeben ihnen und den nunmehr hinzugestoßenen Teilen der Informatiker das gesamte Forschungs- und Entwicklungsfeld? Für Henrichs ist es auf keinem Fall hinzunehmen, die Disziplinen getrennt zu verfolgen, sondern im Gegenteil „den Graben“ zwischen dem Bibliotheks- und Archivwesen einerseits und dem Fachinformationswesen andererseits „weiter zu verfüllen“ (Henrichs, 2014, 285). Auch zwischen Informatik und Informationswissenschaft gibt es Anknüpfungspunkte, wobei die Computerwissenschaft eher für die Technik und die Informationswissenschaft eher für die Inhalte, deren Verstehen und ihre Auswirkungen auf die Menschen zuständig ist. „Mir ging es um das Verstehen der Inhalte; die Technik hat mich eigentlich nur am Rande interessiert“ (Henrichs, 2012, 14). 
Im Gegensatz zur Institutionalisierung der GID war Henrichs bei der Etablierung der Informationswissenschaft an der Heinrich-Heine-Universität Düsseldorf ausgesprochen erfolgreich. Die Magister-Studierenden der Philosophischen Fakultät erhielten die Möglichkeit, Informationswissenschaft als Nebenfach zu studieren, was ihre Chancen auf dem Arbeitsmarkt beträchtlich erhöhte. Henrichs übergab seinem Nachfolger rund 1.000 Studierende, was durchaus als Indikator auf die Beliebtheit des Faches interpretiert werden kann (diese Zahl konnte - mit neuen, veränderten Studiengängen - bis heute erhalten werden). Auf ein entscheidendes Problem wies Henrichs bereits im Jahr 2005 hin: „Die ,Ein-Mann-Show' hat in der Hochschullehre jedenfalls keine Zukunft mehr“" (Henrichs, 2014, 214). Eine Verzahnung mit anderen Fächern an der Philosophischen Fakultät erwies sich in der Tat als schwer realisierbar; zudem war es der Informationswissenschaft nicht gelungen, sich ausreichend durch DFG-Mittel bzw. Sonderforschungsbereiche auszuweisen. Die Fakultät wird deshalb die informationswissenschaftliche Forschung und Lehre in Düsseldorf im Jahr 2022 einstellen.

Letztendlich war Henrichs für die Zukunft der Wissensorganisation optimistisch: „Die gesamte Menschheitsgeschichte kann man als Erfolgsgeschichte der Wissensverarbeitung begreifen - jeweils mit dem Problem des Missbrauchs behaftet. Die Menschheit ist stets besser geworden darin, sich die Welt informationell anzueignen. Information ist der Schlüssel zur Bemächtigung der Welt“" (Henrichs, 2000, 35).

Wir wollen diesen Nachruf mit einigen Aussagen zum Menschen Norbert Henrichs (Abbildung 9) schließen - in seinen Rollen als Nachbar, Chef, Lehrer und Kollege. Vogel und Wirtz $(2000,353)$ beschreiben den Nachbarn Henrichs: „Seine Ansichten bringt er immer wieder auf einige Punkte, die Würde des Menschen und die Freiheit des Gewissens, dies in Verantwortung für den Mitmenschen. Diese Maßstäbe bilden mit anderen das Koordinatensystem seiner Werte“. Und: „Jedem, der zum ersten Mal mit ihm zu tun hat, fällt seine Einfachheit, seine Bescheidenheit auf, die kein gespieltes Understatement ist, sondern sein Lebensstil aus Überzeugung“.

Wolf Rauch (persönliche Mitteilung, 31.5.2016) erinnert sich an den Kollegen und Informationswissenschaftler: „Die Informationswissenschaft hat viele Gesichter. Norbert Henrichs hat die nachdenkliche, besonnene und von ethischen Grundsätzen getragene Seite unseres Faches geprägt wie kein anderer: an der Universität, an der GID und im persönlichen Kontakt. Er war damit Pionier auf einem Gebiet, das für Erfolg oder Misserfolg der Informationsgesellschaft entscheidend sein wird. Hoffen wir, dass viele seinem Weg folgen werden“. Auch seine langjährige Sekretärin Anneliese Volkmar möchte den Informationswissenschaftler nicht vom Menschen Henrichs getrennt sehen (persönliche Mitteilung, 31.5.2016). „Schon beim ersten Gespräch ist mir sein Enthusiasmus für das Fach aufgefallen. Die tägliche Arbeit war geprägt von menschlichem Miteinander, von seinem informationspolitischen und gesellschaftlichen Engagement. Neben Lehre und Forschung war er stets bestrebt, die Welt etwas besser und gerechter zu gestalten; dafür hat er viel Zeit geopfert. Sein Denken ,Der Computer soll den Menschen nicht ersetzen, sondern ihm die Arbeit erleichtern', war mir gleichzeitig Zuspruch und Ansporn für meine Arbeit mit den neuesten Computertechniken. Meine Verbundenheit mit der Informationswissenschaft ist nicht zuletzt seinem Eifer und seinem Einsatz für diese Wissenschaft und deren Umfeld geschuldet“.

Violeta Trkulja und Jasmin Schmitz beschreiben Henrichs als Hochschullehrer (persönliche Mitteilung, 6.6.2016): „Mit seinen heute eher antiquiert wirkenden Overhead-Foliensätzen, seiner Redegewandtheit und Begeisterung vermochte es Norbert Henrichs in seinen Vorlesungen den Studierenden das Gefühl zu geben, bei der Umsetzung des IuD-Programmes in den 1970er Jahren selbst dabei gewesen zu sein. Er war in der Lage nicht nur die neuesten (Er-)Kenntnisse über das damals noch brandneue ,WWW' zu vermitteln, sondern verstand es auch, die Funktionsweise des Videotextes oder den Beschreibungsvorgang einer CD-ROM so zu erläutern, dass sie bis heute im Gedächtnis geblieben sind. Sein theologisches und philosophisches Wissen, seine Menschlichkeit und sein soziales Engagement durchdrangen seine Vorlesungen und Seminare und bereicherten jede Diskussion. Wie kaum ein anderer vermochte er es, Querbezüge zu anderen Themenfeldern und Wissensgebieten über die Informationswissenschaft hinaus herzustellen“. Dirk Lewandowski (persönliche Mitteilung, 2.6.2016) war zwischen 1997 und 2001 Student bei Henrichs. „Ich war beeindruckt davon, dass Professor Henrichs uns Studierende im besten Sinne ernst nahm, ohne dabei in die Gefahr zu geraten, sich anzubiedern oder ein Verhältnis auf gleicher Ebene vorzutäuschen. Er gab uns Anleitung und Anregung für eigenständige Arbeiten, war Diskussionspartner und Lenker - ein echter Lehrender eben. Professor Henrichs hat uns Studierenden gezeigt, dass es mehr gibt als die Sichtweise des eigenen Fachs und innerhalb der Vorlesung die eng begrenzten Inhalte. Ich erinnere mich an viele Diskussionen im Seminar, die für mich informationswissenschaftlich im besten Sinne waren: über die Beziehung von Mensch, In- 
formation und Technik, stets das Zusammenspiel aller drei Elemente im Auge behaltend. Durch seine Art und seinen Anspruch hat Herr Henrichs eine besondere Atmosphäre in der Informationswissenschaft in Düsseldorf erzeugt die des inhärent motivierten, gemeinsamen Lernens zwischen Studierenden und Dozenten“.

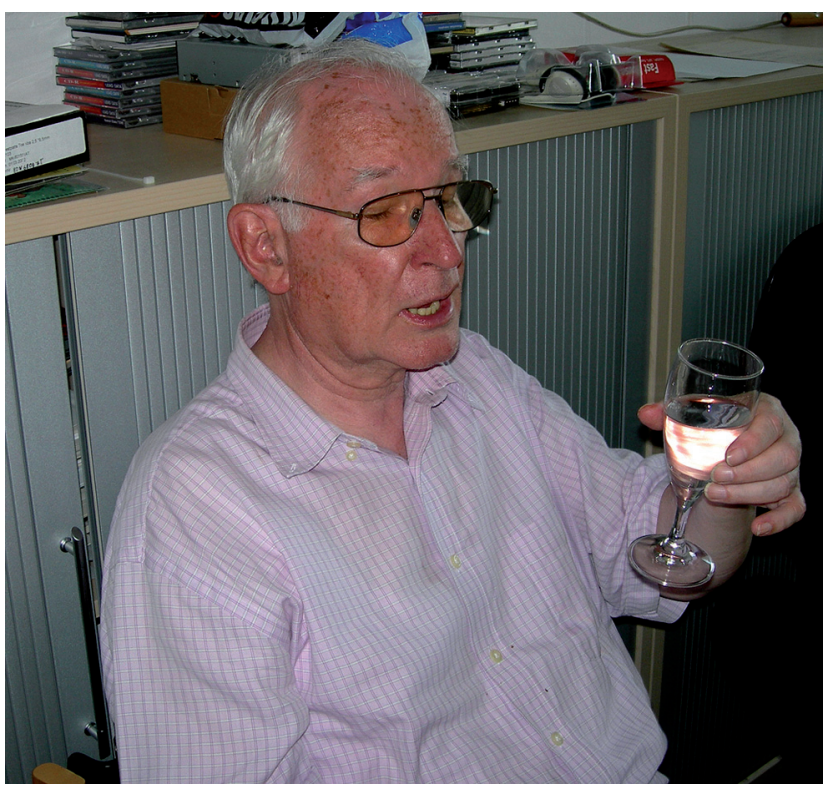

Abbildung 9: Henrichs bei einer Promotionsfeier in Düsseldorf im Juli 2006 (Foto: W.G. Stock).

\section{Literatur}

Anonym (1969). Golem melkt. DER SPIEGEL, Nr. 44, 193-194.

Anonym (1987). Endgültig Schluss. DER SPIEGEL, Nr. 27, 42-43.

Belke, F. (1973). Gesamtregister der Annalen der Philosophie (u. philos. Kritik) 1-8 (1919-1929), Erkenntnis 1-7 (1930/31-1937/38), The Journal of Unified Science (Erkenntnis) 8(1939/40), Forum philosophicum 1 (1930/31).Nendeln, Liechtenstein: Kraus Reprint.

BMFT (1974). Programm der Bundesregierung zur Förderung der Information und Dokumentation 1974-1977. Bonn: Der Bundesminister für Forschung und Technologie.

BMFT (1990). Fachinformationsprogramm der Bundesregierung 1990-1994. Bonn: Der Bundesminister für Forschung und Technologie.

Hauk, K. \& Stock, W.G. (2012).Pioneers of information science in Europe: The Fuvre of Norbert Henrichs. In International Perspectives on the History of Information Science and Technology (pp. 151-162).Medford, NJ: Information Today.

Henrichs, N. (1967). Kult und Brauchtum im Kirchenjahr: Eine kulttheologische und brauchtumsgeschichtliche Untersuchung für Schule und Seelsorge. Düsseldorf: Patmos.

Henrichs, N. (1968). Bibliographie der Hermeneutik und ihrer Anwendungsbereiche seit Schleiermacher. Düsseldorf: Philosophia.

Henrichs, N. (1973). Erläuterungen zum Inhalt, Aufbau und zur Benutzung der Bibliographie. In F. Belke, Gesamtregister der Annalen der Philosophie (u. philos. Kritik) 1-8 (1919-1929), Erkenntnis 1-7 (1930/31-1937/38), The Journal of Unified Science (Erkenntnis) 8 (1939/40), Forum philosophicum 1 (1930/31) (pp. VII-IX). Nendeln, Liechtenstein: Kraus Reprint.

Henrichs, N. (1990). Informationswissenschaft in Düsseldorf. In M. Buder, W. Rehfeld, \& T. Seeger (Hrsg.), Grundlagen derpraktischen Information und Dokumentation. 3. Aufl. (pp.1062-1072). München: Saur.

Henrichs, N. (2000). Konsens und Vertrauen als Regularien. Password, Nr. 7/8, 35.

Henrichs, N. (2007). In Memoriam. Nachruf auf Ministerialrat a. D. Dr. Heinz Lechmann. Information - Wissenschaft und Praxis, 58(7), 380-381.

Henrichs, N. (2012). Oral-History-Interview mit Norbert Henrichs am 12. April 2012 in Düsseldorf. Interviewer: W.G. Stock, Protokoll: A. Volkmar und K. Hauk, Video: L. Beutelspacher. Düsseldorf: Abteilung für Informationswissenschaft (unveröffentl. Video und Transkript).

Henrichs, N. (2014). Menschsein im Informationszeitalter. Informationswissenschaft mit Leidenschaft und missionarischem Eifer. Glückstadt: Hülsbusch.

Henrichs, N., Mayer, W. \& Menges, G., Hrsg. (1995). Kaiserswerther Vorträge zu Friedrich Spee 1985-1993. Düsseldorf: Heimat- und Bürgerverein Kaiserswerth.

Henrichs, N. \& Weeland, H., Hrsg. (1987). Briefwechsel deutschsprachiger Philosophen 1750-1850. München: Saur.

Henrichs, N. \& Weeland, H., Hrsg. (1990). Briefe Deutscher Philosophen (1750-1850).München: Saur.

Kuhlen, R. (2014). Geleitwort. In N. Henrichs, Menschsein im Informationszeitalter. Informationswissenschaft mit Leidenschaft und missionarischem Eifer (p. v). Glückstadt: Hülsbusch.

Linde, F. \& Stock, W.G. (2011). Informationsmarkt. Informationen im ICommerce anbieten und nachfragen. München: Oldenbourg.

Ockenfeld, M. (2016). Menschsein im Informationszeitalter. Informationswissenschaft mit Leidenschaft und missionarischem Eifer [Rezension]. Information - Wissenschaft und Praxis, 67(2-3), 159-161.

Rauch, W. (2000). Auf dem Weg zur Informationskultur. Meilensteine des Paradigmenwechsels. In T.A. Schröder (Hrsg.), Aufdem Weg zur Informationskultur. Wa(h)re Information? Festschrift für Norbert Henrichs zum 65. Geburtstag (pp. 25-30). Düsseldorf: Universitäts- und Landesbibliothek.

Schröder, T.A., Hrsg. (2000). Auf dem Weg zur Informationskultur. Wa(h)re Information? Festschrift für Norbert Henrichs zum 65. Geburtstag. Düsseldorf: Universitäts- und Landesbibliothek. Stock, W.G. (1985). Empirische Philosophieforschung. Informetrische Ansätze zur quantitativen Bestimmung philosophischer Thematiken als Teil einer empirischen Metaphilosophie. Zeitschrift für philosophische Forschung, 39, 431-455.

Stock, W.G. (2000). Textwortmethode. Password, Nr. 7/8, 26-35. Stock, W.G. \& Stock, M. (2013). Handbook of Information Science. Berlin, Boston, MA: De Gruyter Saur.

Vogels, F.J. \& Wirtz, P. (2000). Zur Emeritierung von Prof. Dr. Norbert Henrichs. In T.A. Schröder (Hrsg.), Aufdem Weg zur Informationskultur. Wa(h)re Information? Festschrift für Norbert Henrichs zum 65. Geburtstag (pp. 353-354). Düsseldorf: Universitätsund Landesbibliothek.

Wissenschaftsrat (1984). Stellungnahme zur Gesellschaft für Information und Dokumentation (GID). Berlin: Wissenschaftsrat. 